\title{
Remarks on inequalities of Hardy-Sobolev Type
}

Ying-Xiong Xiao

Correspondence: yxxiao.

math@gmail.com

School of Mathematics and

Statistics, Xiaogan University,

Xiaogan 432000 Hubei, People's

Republic of China

\section{Abstract}

We obtain the sharp constants of some Hardy-Sobolev-type inequalities proved by Balinsky et al. (Banach J Math Anal 2(2):94-106).

2000 Mathematics Subject Classification: Primary 26D10; $46 E 35$.

Keywords: Hardy inequality, Sobolev Inequality

\section{Introduction}

Hardy inequality in $\mathbb{R}^{n}$ reads, for all $f \in C_{0}^{\infty}\left(\mathbb{R}^{n}\right)$ and $n \geq 3$,

$$
\int_{\mathbb{R}^{n}}|\nabla f|^{2} \mathrm{~d} x \geq \frac{(n-2)^{2}}{4} \int_{\mathbb{R}^{n}} \frac{f^{2}}{|x|^{2}} \mathrm{~d} x .
$$

The Sobolev inequality states that, for all $f \in C_{0}^{\infty}\left(\mathbb{R}^{n}\right)$ and $n \geq 3$,

$$
\int_{\mathbb{R}^{n}}|\nabla f|^{2} \mathrm{~d} x \geq S_{n}\left(\int_{\mathbb{R}^{n}}|f|^{2^{*}} \mathrm{~d} x\right)^{\frac{2}{2^{*}}},
$$

where $2^{*}=\frac{2 n}{n-2}$ and $S_{n}=\pi n(n-2)\left(\Gamma\left(\frac{n}{2}\right) / \Gamma(n)\right)^{\frac{2}{n}}$ is the best constant (cf. [1,2]). A result of Stubbe [3] states that for $0 \leq \delta<\frac{(n-2)^{2}}{4}$,

$$
\left.\int_{\mathbb{R}^{n}}|\nabla f|^{2} \mathrm{~d} x-\delta \int_{\mathbb{R}^{n}} \frac{f^{2}}{|x|^{2}} \mathrm{~d} x \geq\left.\frac{\left(\frac{(n-2)^{2}}{4}-\delta\right)^{\frac{n-1}{n}}}{\left(\frac{(n-2)^{2}}{4}\right)^{\frac{n-1}{n}}} S_{n}\left|\int_{\mathbb{R}^{n}}\right| f\right|^{2^{*}} \mathrm{~d} x\right)^{\frac{2}{2^{*}}}
$$

and the constant in (1.3) is sharp. Recently, Balinsky et al. [4] prove analogous inequalities for the operator $\mathcal{L}:=\mathbf{x} \cdot \nabla$. One of the results states that, for $0 \leq \delta<n^{2} / 4$ and $f \in C_{0}^{\infty}\left(\mathbb{R}^{n}\right)$,

$$
\int_{\mathbb{R}^{n}}|\mathcal{L} f|^{2} \mathrm{~d} x-\delta \int_{\mathbb{R}^{n}} f^{2} \mathrm{~d} x \geq C\left(\frac{n^{2}}{4}-\delta\right)^{\frac{n-1}{n}} S_{n}\left(\int_{\mathbb{R}^{n}}|r F|^{2^{*}} \mathrm{~d} x\right)^{\frac{2}{2^{*}}},
$$


where $F(r)$ is the integral mean of $f$ over the unit sphere $\mathbb{S}^{n-1}$, i.e.,

$$
F(r)=\frac{1}{\left|\mathbb{S}^{n-1}\right|} \int_{\mathbb{S}^{n-1}} f(r \omega) \mathrm{d} \omega,
$$

and $\left|\mathbb{S}^{n-1}\right|=\int_{\mathbb{S}^{n-1}} d \omega=\frac{2 \pi^{n / 2}}{\Gamma(n / 2)}$. Here, we use the polar coordinates $x=r \omega$. The aim of this note is to look for the sharp constant of inequality (1.4). To this end, we have:

Theorem 1.1. Let $f \in C_{0}^{\infty}\left(\mathbb{R}^{n}\right)$ and $n \geq 3$. There holds, for $0 \leq \delta<\frac{n^{2}}{4}$,

$$
\int_{\mathbb{R}^{n}}|\mathcal{L} f|^{2} \mathrm{~d} x-\delta \int_{\mathbb{R}^{n}} f^{2} \mathrm{~d} x \geq \frac{\left(\frac{n^{2}}{4}-\delta\right)^{\frac{n-1}{n}}}{\left(\frac{(n-2)^{2}}{4}\right)^{\frac{n-1}{n}}} S_{n}\left(\int_{\mathbb{R}^{n}}|r F(r)|^{2^{*}} \mathrm{~d} x\right)^{\frac{2}{2^{*}}}
$$

and the constant in (1.5) is sharp.

When $\delta=n^{2} / 4$, we have the following Theorem, which generalize the results of [4], Corollary 4.6.

Theorem 1.2. If $f$ is supported in the annulus $A_{R}:=\left\{x \in \mathbb{R}^{n}: R^{-1}<|x|<R\right\}$, then

$$
\int_{A_{R}}|\mathcal{L} f|^{2} \mathrm{~d} x-\frac{n^{2}}{4} \int_{A_{R}} f^{2} \mathrm{~d} x \geq[2(n-2) \ln R]^{-\frac{2(n-1)}{n}} S_{n}\left(\int_{A_{R}}|r F(r)|^{2^{*}} \mathrm{~d} x\right)^{\frac{2}{2^{*}}} .
$$

\section{The proofs}

We first recall the Bliss lemma [5]:

Lemma 2.1. For $s \geq 0, q>p>1$ and $r=q / p-1$,

$$
\left(\int_{0}^{\infty}\left|\int_{0}^{s} g(t) \mathrm{d} t\right|^{q} s^{r-q} \mathrm{~d} s\right)^{p / q} \leq C_{p, q} \int_{0}^{\infty}|g(t)|^{p} \mathrm{~d} t
$$

where

$$
C_{p, q}=(q-r-1)^{-p / q}\left(\frac{r \Gamma(q / r)}{\Gamma(1 / r) \Gamma((q-1) / r)}\right)^{r p / q}
$$

is the sharp constant. Equality is attained for functions of the form

$$
g(t)=c_{1}\left(c_{2} s^{r}+1\right)^{-\frac{r+1}{r}}, \quad c_{1}>0, c_{2}>0 .
$$

Using the Bliss lemma, we can prove the Theorem 1.1 for the radial function $f$, i.e., $f(x)=\tilde{f}(|x|)$ for some $\tilde{f} \in C_{0}^{\infty}([0, \infty))$. 
Lemma 2.2. Let $f(|x|) \in C_{0}^{\infty}\left(\mathbb{R}^{n}\right)$ and $n \geq 3$. There holds, for $0 \leq \delta<\frac{n^{2}}{4}$,

$$
\int_{\mathbb{R}^{n}}|\mathcal{L} f|^{2} \mathrm{~d} x-\delta \int_{\mathbb{R}^{n}} f^{2} \mathrm{~d} x \geq \frac{\left(\frac{n^{2}}{4}-\delta\right)^{\frac{n-1}{n}}}{\left(\frac{(n-2)^{2}}{4}\right)^{\frac{n-1}{n}}} S_{n}\left(\int_{\mathbb{R}^{n}}|r F(r)|^{2^{*}} \mathrm{~d} x\right)^{\frac{2}{2^{*}}}
$$

and the constant in (2.1) is sharp.

Proof. We note if $f$ is radial, then $F(r)=f(r)$ and $\mathcal{L} f=r f^{\prime}(r)$. Therefore, inequality (2.1) is equivalent to

$$
\begin{aligned}
& \int_{0}^{\infty}\left|f^{\prime}(r)\right|^{2} r^{n+1} \mathrm{~d} r-\delta \int_{0}^{\infty}|f(r)|^{2} r^{n-1} \mathrm{~d} r \\
& \geq \frac{\left(\frac{n^{2}}{4}-\delta\right)^{\frac{n-1}{n}}}{\left(\frac{(n-2)^{2}}{4}\right)^{\frac{n-1}{n}}} S_{n} \cdot\left|\mathbb{S}^{n-1}\right|-\frac{2}{n}\left(\int_{0}^{\infty}|f(r)|^{2^{*}} r^{2^{*}+n-1} \mathrm{~d} x\right)^{\frac{2}{2^{*}}} .
\end{aligned}
$$

Let $0 \leq \beta<n / 2$ and set $g(r)=r^{\beta} f(r)$. Through integration by parts, we have that

$$
\int_{0}^{\infty}\left|g^{\prime}(r)\right|^{2} r^{n+1-2 \beta} \mathrm{d} r=\int_{0}^{\infty}\left|f^{\prime}(r)\right|^{2} r^{n+1} \mathrm{~d} r-\beta(n-\beta) \int_{0}^{\infty}|f(r)|^{2} r^{n-1} \mathrm{~d} r .
$$

Make the change of variables $s=r^{n-2 \beta}$,

$$
\int_{0}^{\infty}\left|g^{\prime}(r)\right|^{2} r^{n+1-2 \beta} \mathrm{d} r=(n-2 \beta) \int_{0}^{\infty} s^{2}\left|\frac{\partial g}{\partial s}\right|^{2} \mathrm{~d} s .
$$

On the other hand, set $h(s)=\frac{\partial g}{\partial s}$ so that $g=-\int_{s}^{+\infty} h(t) \mathrm{d} t$, we have

$$
\int_{0}^{\infty} s^{2}\left|\frac{\partial g}{\partial s}\right|^{2} \mathrm{~d} s=\int_{0}^{\infty} s^{2} h^{2} \mathrm{~d} s=\int_{0}^{\infty}|w(s)|^{2} \mathrm{~d} s
$$

where $w(s)=s^{-2} h\left(s^{-1}\right)$. By Bliss lemma,

$$
\int_{0}^{\infty}|w(s)|^{2} \mathrm{~d} s \geq\left(\frac{n}{n-2}\right)^{\frac{n-2}{n}}\left(\frac{\Gamma(n / 2) \Gamma(1+n / 2)}{\Gamma(n)}\right)^{\frac{2}{n}}\left(\int_{0}^{\infty}\left|\int_{0}^{s}\right| w(t)|\mathrm{d} t|^{2^{*}} \frac{2-2 n}{s-2} \mathrm{~d} s\right)^{\frac{2}{2^{*}}},
$$


Xiao Journal of Inequalities and Applications 2011, 2011:132

Page 4 of 8

http://www.journalofinequalitiesandapplications.com/content/2011/1/132

i.e.,

$$
\begin{aligned}
& \int_{0}^{\infty} s^{2}\left|\frac{\partial g}{\partial s}\right|^{2} \mathrm{~d} s=\int_{0}^{\infty} s^{2} h^{2} \mathrm{~d} s=\int_{0}^{\infty}|w(s)|^{2} \mathrm{~d} s \\
& \geq\left(\frac{n}{n-2}\right)^{\frac{n-2}{n}}\left(\frac{\Gamma(n / 2) \Gamma(1+n / 2)}{\Gamma(n)}\right)^{\frac{2}{n}}\left(\int_{0}^{\infty}\left|\int_{0}^{s}\right| w(t)|\mathrm{d} t|^{2^{*}} \frac{2-2 n}{n-2} \mathrm{~d} s\right)^{\frac{2}{2^{*}}} \\
& =\left(\frac{n}{n-2}\right)^{\frac{n-2}{n}}\left(\frac{\Gamma(n / 2) \Gamma(1+n / 2)}{\Gamma(n)}\right)^{\frac{2}{n}}\left(\int_{0}^{\infty}\left|\int_{s}^{+\infty}\right| h(t)|\mathrm{d} t|^{2^{*}} \frac{2}{s-2} \mathrm{~d} s\right)^{\frac{2}{2^{*}}} \\
& \geq\left(\frac{n}{n-2}\right)^{\frac{n-2}{n}}\left(\frac{\Gamma(n / 2) \Gamma(1+n / 2)}{\Gamma(n)}\right)^{\frac{2}{n}}\left(\int_{0}^{\infty}|g|^{2^{*}} \frac{2}{n-2} \mathrm{~d} s\right)^{\frac{2}{2^{*}}} \cdot
\end{aligned}
$$

Recall that $s=r^{n-2 \beta}$ and $g(r)=r^{\beta} f(r)$,

$$
\int_{0}^{\infty} g^{2^{*}}{ }_{s} \frac{2}{n-2} \mathrm{~d} s=(n-2 \beta) \int_{0}^{\infty}\left(r^{1-\beta} g\right)^{2^{*}} r^{n-1} \mathrm{~d} r=(n-2 \beta) \int_{0}^{\infty}(r f)^{2^{*}} r^{n-1} \mathrm{~d} r .
$$

Therefore, by (2.3), (2.4), (2.5) and (2.6),

$$
\begin{aligned}
& \int_{0}^{\infty}\left|f^{\prime}(r)\right|^{2} r^{n+1} \mathrm{~d} r-\beta(n-\beta) \int_{0}^{\infty}|f(r)|^{2} r^{n-1} \mathrm{~d} r \\
& =(n-2 \beta) \int_{0}^{\infty} s^{2}\left|\frac{\partial g}{\partial s}\right|^{2} \mathrm{~d} s \\
& \geq(n-2 \beta)^{1+\frac{2}{2^{*}}}\left(\frac{n}{n-2}\right)^{\frac{n-2}{n}}\left(\frac{\Gamma(n / 2) \Gamma(1+n / 2)}{\Gamma(n)}\right)^{\frac{2}{n}}\left(\int_{0}^{\infty}(r f)^{\left.2^{*} r^{n-1} \mathrm{~d} r\right)^{\frac{2}{2^{*}}}}\right. \\
& =(n-2 \beta)^{\frac{2 n-2}{n}}\left(\frac{n}{n-2}\right)^{\frac{n-2}{n}}\left(\frac{\Gamma(n / 2) \Gamma(1+n / 2)}{\Gamma(n)}\right)^{\frac{2}{n}}\left(\int_{0}^{\infty}(r f)^{2^{*}} r^{n-1} \mathrm{~d} r\right)^{\frac{2}{2^{*}}} .
\end{aligned}
$$

Since $\left|\mathbb{S}^{n-1}\right|=\int_{\mathbb{S}^{n-1}} \mathrm{~d} \omega=\frac{2 \pi^{n / 2}}{\Gamma(n / 2)}$ and $S_{n}=\pi n(n-2)\left(\Gamma\left(\frac{n}{2}\right) / \Gamma(n)\right)^{\frac{2}{n}}$, we have

$$
\begin{aligned}
& \int_{\mathbb{R}^{n}}|\mathcal{L} f|^{2} \mathrm{~d} x-\beta(n-\beta) \int_{\mathbb{R}^{n}} f^{2} \mathrm{~d} x \\
& =\left|\mathbb{S}^{n-1}\right| \int_{0}^{\infty}\left|f^{\prime}(r)\right|^{2} r^{n+1} \mathrm{~d} r-\beta(n-\beta)\left|\mathbb{S}^{n-1}\right| \int_{0}^{\infty}|f(r)|^{2} r^{n-1} \mathrm{~d} r \\
& \geq\left|\mathbb{S}^{n-1}\right| \cdot(n-2 \beta) \frac{2 n-2}{n}\left(\frac{n}{n-2}\right)^{\frac{n-2}{n}}\left(\frac{\Gamma(n / 2) \Gamma(1+n / 2)}{\Gamma(n)}\right)^{\frac{2}{n}}\left(\int_{0}^{\infty}(r f)^{2^{*}} r^{n-1} \mathrm{~d} r\right)^{\frac{2}{2^{*}}} \\
& =\left|\mathbb{S}^{n-1}\right|^{\frac{2}{n}}(n-2 \beta) \frac{2 n-2}{n}\left(\frac{n}{n-2}\right)^{\frac{n-2}{n}}\left(\frac{\Gamma(n / 2) \Gamma(1+n / 2)}{\Gamma(n)}\right)^{\frac{2}{n}}\left(\int_{\mathbb{R}^{n}}|r f(r)|^{2^{*}} \mathrm{~d} x\right)^{\frac{2}{2^{*}}} \\
& =\left(\frac{n-2 \beta}{n-2}\right)^{\frac{2 n-2}{n}} S_{n}\left(\int_{\mathbb{R}^{n}}|r f(r)|^{2^{*}} \mathrm{~d} x\right)^{\frac{2}{2^{*}}} .
\end{aligned}
$$


Xiao Journal of Inequalities and Applications 2011, 2011:132

Page 5 of 8

http://www.journalofinequalitiesandapplications.com/content/2011/1/132

Let $\beta=\frac{n-\sqrt{n^{2}-4 \delta}}{2}$ when $0 \leq \delta<n^{2} / 4$. Then, $0 \leq \beta<n / 2$ and $\delta=\beta(n-\beta)$. Therefore,

$$
\int_{\mathbb{R}^{n}}|\mathcal{L} f|^{2} \mathrm{~d} x-\delta \int_{\mathbb{R}^{n}} f^{2} \mathrm{~d} x \geq\left(\frac{n^{2}-4 \delta}{(n-2)^{2}}\right)^{\frac{n-1}{n}} S_{n}\left(\int_{\mathbb{R}^{n}}|r f(r)|^{2^{*}} \mathrm{~d} x\right)^{\frac{2}{2^{*}}} .
$$

Inequality (2.1) follows.

Now we can prove Theorem 1.1.

Proof of Theorem 1.1. Decomposing $f$ into spherical harmonics, we get (see e.g. [6])

$$
f=\sum_{k=0}^{\infty} f_{k}:=\sum_{k=0}^{\infty} g_{k}(r) \phi_{k}(\sigma)
$$

where $\varphi_{k}(\sigma)$ are the orthonormal eigenfunction of the Laplace-Beltrami operator with responding eigenvalues

$$
c_{k}=k(N+k-2), \quad k \geq 0 .
$$

The functions $g_{k}(r)$ belong to $C_{0}^{\infty}\left(\mathbb{R}^{n}\right)$, satisfying $g_{k}(r)=O\left(r^{k}\right)$ and $g_{k}^{\prime}(r)=O\left(r^{k-1}\right)$ as $r$ $\rightarrow 0$. By orthogonality,

$$
F(r)=\frac{1}{\left|\mathbb{S}^{n-1}\right|} \int_{\mathbb{S}^{n-1}} f(r \omega) \mathrm{d} \omega=g_{0}(r)
$$

On the other hand,

$$
\mathcal{L} f(x)=\sum_{k=0}^{\infty} r \frac{\partial\left(g_{k}(r) \phi_{k}\right)}{\partial r}=\sum_{k=0}^{\infty} r g_{k}^{\prime}(r) \phi_{k}(\sigma) .
$$

Here, we use the radial derivative $\frac{\partial}{\partial r}=\frac{\mathbf{x} \cdot \nabla}{|x|}=\frac{\mathcal{L}}{|x|}$. Therefore,

$$
\begin{aligned}
& \int_{\mathbb{R}^{n}}|\mathcal{L} f|^{2} \mathrm{~d} x-\delta \int_{\mathbb{R}^{n}} f^{2} \mathrm{~d} x=\sum_{k=0}^{\infty}\left(\int_{\mathbb{R}^{n}} r^{2}\left|g^{\prime}{ }_{k}(r)\right|^{2} \mathrm{~d} x-\delta \int_{\mathbb{R}^{n}} g_{k}^{2} \mathrm{~d} x\right) \\
& \geq \int_{\mathbb{R}^{n}} r^{2}\left|g^{\prime}{ }_{0}(r)\right|^{2} \mathrm{~d} x-\delta \int_{\mathbb{R}^{n}} g_{0}^{2} \mathrm{~d} x=\int_{\mathbb{R}^{n}} r^{2}\left|F^{\prime}(r)\right|^{2} \mathrm{~d} x-\delta \int_{\mathbb{R}^{n}} F(r)^{2} \mathrm{~d} x
\end{aligned}
$$

since

$$
\int_{\mathbb{R}^{n}}|\mathcal{L} u|^{2} \mathrm{~d} x \geq \frac{n^{2}}{4} \int_{\mathbb{R}^{n}} u^{2} \mathrm{~d} x
$$


holds for all $u \in C_{0}^{\infty}\left(\mathbb{R}^{n}\right)$ and $\mathcal{L} u=r u^{\prime}(r)$ if $u$ is radial. By Lemma 2.2,

$$
\int_{\mathbb{R}^{n}} r^{2}\left|F^{\prime}(r)\right|^{2} \mathrm{~d} x-\delta \int_{\mathbb{R}^{n}} F(r)^{2} \mathrm{~d} x \geq \frac{\left(\frac{n^{2}}{4}-\delta\right)^{\frac{n-1}{n}}}{\left(\frac{(n-2)^{2}}{4}\right)^{\frac{n-1}{n}}} S_{n}\left(\int_{\mathbb{R}^{n}}|r F(r)|^{2^{*}} \mathrm{~d} x\right)^{\frac{2}{2^{*}}}
$$

Therefore,

$$
\begin{aligned}
& \int_{\mathbb{R}^{n}}|\mathcal{L} f|^{2} \mathrm{~d} x-\delta \int_{\mathbb{R}^{n}} f^{2} \mathrm{~d} x \\
& \geq \int_{\mathbb{R}^{n}} r^{2}\left|F^{\prime}(r)\right|^{2} \mathrm{~d} x-\delta \int_{\mathbb{R}^{n}} F(r)^{2} \mathrm{~d} x \\
& \geq \frac{\left(\frac{n^{2}}{4}-\delta\right)^{\frac{n-1}{n}}}{\left(\frac{(n-2)^{2}}{4}\right)^{\frac{n-1}{n}}} S_{n}\left(\int_{\mathbb{R}^{n}}|r F(r)|^{2^{*}} \mathrm{~d} x\right)^{\frac{2}{2^{*}}} .
\end{aligned}
$$

The proof of Theorem 1.1 is completed.

Proof of Theorem 1.2. We denote by $B_{R} \subset \mathbb{R}^{N}$ the unit ball centered at zero.

Step 1. Assume $f$ is radial and $f \in C_{0}^{\infty}\left(B_{R}\right)$. Then,

$$
\begin{aligned}
& \int_{B_{R}}|\mathcal{L} f|^{2} \mathrm{~d} x-\frac{n^{2}}{4} \int_{B_{R}} f^{2} \mathrm{~d} x=\int_{B_{R}}\left|r f^{\prime}(r)\right|^{2} \mathrm{~d} x-\frac{n^{2}}{4} \int_{B_{R}} f^{2}(r) \mathrm{d} x \\
& =\int_{B_{R}}\left|(r f(r))^{\prime}\right|^{2} \mathrm{~d} x-\frac{(n-2)^{2}}{4} \int_{B_{R}} \frac{(r f)^{2}}{|x|^{2}} \mathrm{~d} x .
\end{aligned}
$$

Therefore, by Theorem B in [7],

$$
\begin{aligned}
& \int_{B_{R}}\left|(r f(r))^{\prime}\right|^{2} \mathrm{~d} x-\frac{(n-2)^{2}}{4} \int_{B_{R}} \frac{(r f)^{2}}{|x|^{2}} \mathrm{~d} x \\
& \geq(n-2)^{-\frac{2(n-1)}{n}} S_{n}\left(\int_{B_{R}} X_{1}^{\frac{2(n-1)}{n-2}}\left(a, \frac{|x|}{R}\right)|r f|^{\frac{2 n}{n-2}} \mathrm{~d} x\right)^{\frac{n-2}{n}},
\end{aligned}
$$

where

$$
X_{1}(a, s):=(a-\ln s)^{-1}, \quad a>0,0<s \leq 1 .
$$


Thus,

$$
\begin{aligned}
& \int_{B_{R}}|\mathcal{L} f|^{2} \mathrm{~d} x-\frac{n^{2}}{4} \int_{B_{R}} f^{2} \mathrm{~d} x=\int_{B_{R}}\left|r f^{\prime}(r)\right|^{2} \mathrm{~d} x-\frac{n^{2}}{4} \int_{B_{R}} f^{2}(r) \mathrm{d} x \\
& \geq(n-2)^{-\frac{2(n-1)}{n}} S_{n}\left(\int_{B_{R}} X_{1} \frac{2(n-1)}{n-2}\left(a, \frac{|x|}{R}\right)|r f|^{\frac{2 n}{n-2}} \mathrm{~d} x\right)^{\frac{n-2}{n}} .
\end{aligned}
$$

Step 2. Assume $f$ is not radial and $f \in C_{0}^{\infty}\left(B_{R}\right)$. We extend $f$ as zero outside $B_{R}$. So $f \in C_{0}^{\infty}\left(\mathbb{R}^{n}\right)$. Decomposing $f$ into spherical harmonics, we have

$$
f=\sum_{k=0}^{\infty} f_{k}:=\sum_{k=0}^{\infty} g_{k}(r) \phi_{k}(\sigma)
$$

where $\varphi_{k}(\sigma)$ are the orthonormal eigenfunctions of the Laplace-Beltrami operator with responding eigenvalues

$$
c_{k}=k(N+k-2), \quad k \geq 0 .
$$

The functions $f_{k}(r)$ belong to $C_{0}^{\infty}\left(B_{R}\right)$. By the proof of Theorem 1.1 and Step 1,

$$
\begin{aligned}
& \int_{\mathbb{R}^{n}}|\mathcal{L} f|^{2} \mathrm{~d} x-\frac{n^{2}}{4} \int_{\mathbb{R}^{n}} f^{2} \mathrm{~d} x=\sum_{k=0}^{\infty}\left(\int_{\mathbb{R}^{n}} r^{2}\left|g_{k}^{\prime}(r)\right|^{2} \mathrm{~d} x-\frac{n^{2}}{4} \int_{\mathbb{R}^{n}} g_{k}^{2} \mathrm{~d} x\right) \\
& \geq \int_{\mathbb{R}^{n}} r^{2}\left|g_{0}^{\prime}(r)\right|^{2} \mathrm{~d} x-\frac{n^{2}}{4} \int_{\mathbb{R}^{n}} g_{0}^{2} \mathrm{~d} x=\int_{\mathbb{R}^{n}} r^{2}\left|F^{\prime}(r)\right|^{2} \mathrm{~d} x-\frac{n^{2}}{4} \int_{\mathbb{R}^{n}} F(r)^{2} \mathrm{~d} x \\
& \geq(n-2)^{-\frac{2(n-1)}{n}} S_{n}\left(\int_{B_{R}} \frac{2(n-1)}{X_{1}}\left(a, \frac{|x|}{R}\right)|r F| \frac{2 n}{n-2} \mathrm{~d} x\right)^{\frac{n-2}{n}} .
\end{aligned}
$$

Step 3. By Step 1 and Step 2, the following inequality holds for $f \in C_{0}^{\infty}\left(B_{R}\right)$

$$
\int_{\mathbb{R}^{n}}|\mathcal{L} f|^{2} \mathrm{~d} x-\frac{n^{2}}{4} \int_{\mathbb{R}^{n}} f^{2} \mathrm{~d} x \geq(n-2)^{-\frac{2(n-1)}{n}} S_{n}\left(\int_{B_{\mathbb{R}}} X_{1}^{\frac{2(n-1)}{n-2}}\left(a, \frac{|x|}{R}\right)|r F|^{\frac{2 n}{n-2}} \mathrm{~d} x\right)^{\frac{n-2}{n}} .
$$

We note if $R^{-1}<|x|<R$, then

$$
X_{1}^{\frac{2(N-1)}{N-2}}\left(a, \frac{|x|}{D}\right)=\left(\frac{1}{a-\ln \frac{|x|}{R}}\right)^{\frac{2(N-1)}{N-2}} \geq\left(\frac{1}{a+2 \ln R}\right)^{\frac{2(N-1)}{N-2}} .
$$


Therefore, If $f$ is supported in the annulus $A_{R}:=\left\{x \in \mathbb{R}^{n}: R^{-1}<|x|<R\right\}$, then

$$
\int_{A_{R}}|\mathcal{L} f|^{2} \mathrm{~d} x-\frac{n^{2}}{4} \int_{A_{R}} f^{2} \mathrm{~d} x \geq[(n-2)(2 \ln R+a)]^{-\frac{2(n-1)}{n}} S_{n}\left(\int_{A_{R}}|r F(r)|^{2^{*}} \mathrm{~d} x\right)^{\frac{2}{2^{*}}} .
$$

Letting $a \rightarrow 0$, we have

$$
\int_{A_{R}}|\mathcal{L} f|^{2} \mathrm{~d} x-\frac{n^{2}}{4} \int_{A_{R}} f^{2} \mathrm{~d} x \geq[2(n-2) \ln R]^{-\frac{2(n-1)}{n}} S_{n}\left(\int_{A_{R}}|r F(r)|^{2^{*}} \mathrm{~d} x\right)^{\frac{2}{2^{*}}} .
$$

The proof of Theorem 2 is completed.

\section{Acknowledgements}

The author thanks the referee for his/her careful reading and very useful comments that improved the final version of this paper.

\section{Authors' contributions}

YX designed and performed all the steps of proof in this research and also wrote the paper. All authors read and approved the final manuscript.

\section{Competing interests}

The authors declare that they have no competing interests.

Received: 15 April 2011 Accepted: 5 December 2011 Published: 5 December 2011

\section{References}

1. Aubin, T: Probléme isopérimétric et espace de Sobolev. J Differ Geom. 11, 573-598 (1976)

2. Talenti, G: Best constant in Sobolev inequality. Ann Matem Pura Appl. 110(4), 353-372 (1976)

3. Stubbe, J: Bounds on the number of bound states for potentials with critical decay at infinity. J Math Phys. 31(5), 1177-1180 (1990). doi:10.1063/1.528750

4. Balinsky, A, Evans, WD, Hundertmark, D, Lewis, RT: On inequalities of Hardy-Sobolev type. Banach J Math Anal. 2(2), 94-106 (2008)

5. Bliss, G: An integral inequality. J Lond Math Soc. 5, 40-46 (1930). doi:10.1112/Jlms/s1-5.1.40

6. Tertikas, A, Zographopoulos, NB: Best constants in the Hardy-Rellich inequalities and related improvements. Adv Math. 209, 407-459 (2007). doi:10.1016/i.aim.2006.05.011

7. Adimurthi, , Filippas, S, Tertikas, A: On the best constant of Hardy-Sobolev inequalities. Nonlinear Anal. 70, 2826-2833 (2009). doi:10.1016/j.na.2008.12.019

doi:10.1186/1029-242X-2011-132

Cite this article as: Xiao: Remarks on inequalities of Hardy-Sobolev Type. Journal of Inequalities and Applications $20112011: 132$.

\section{Submit your manuscript to a SpringerOpen ${ }^{\circ}$ journal and benefit from:}

- Convenient online submission

- Rigorous peer review

- Immediate publication on acceptance

- Open access: articles freely available online

- High visibility within the field

- Retaining the copyright to your article

Submit your next manuscript at $>$ springeropen.com 